\title{
-NOTES-
}

\section{SINGULARITY AT THE APEX OF PYRAMIDAL NOTCHES WITH THREE EQUAL ANGLES*}

\author{
BY L. M. KEER AND K. S. PARIHAR** (Northwestern University, Evanston) \\ Northwestern University, Evanston
}

\begin{abstract}
A Green's function approach is employed to study the potential theory problem of determining the strength of singularity at the apex of a pyramid with three equal angles. The problem is reduced to finding the eigenvalue of a singular integral equation. Numerical results are obtained and compared with available literature.
\end{abstract}

Introduction. The potential theory problem of determining the strength of the singularity at the apex of a pyramid with three equal angles has been studied by Bažant [1]. The problem studied there was that of a harmonic function in a region with a pyramidal boundary, with constant potential on the boundary. He developed a solution by use of a general numerical (finite-difference) scheme for potential theory in which several lines of singularity intersect. In particular, he found that when the pyramidal boundary was reentrant to the space considered, the potential behavior with distance was $O\left(\rho^{\gamma}\right)(0 \leq \gamma \leq$ 1) as $\rho \rightarrow 0$, where $\rho$ is the distance to the pyramidal apex. This means that the gradient of the potential (flux) will have a singularity $O\left(\rho^{\gamma-1}\right)$ as $\rho \rightarrow 0$. However, if the pyramid is not reentrant, the paramenter $\gamma$ will be greater than unity and will not produce any singularities in the flux.

In the present study a Green's function method is employed to examine the title problem. An advantage with the proposed method is that for a prescribed accuracy less numerical effort is required than with the finite-difference scheme [1], although more analysis is required to develop the equations into a form suitable for the numerical analysis. Similar results have been noted in the authors' earlier investigation [2] of the problem of determining the strength of singularity at the corner of a wedge-shaped region.

Formulation of the problem. Consider the pyramid $O A B C$ with the three edges $O A$, $O B$, and $O C$ oriented in such a way that the angle between any two consecutive edges is the same, say, $2 \beta$ (where $\beta<\pi / 3$ ). The points $A, B$ and $C$ are taken anywhere on the semiinfinite edges of the pyramid with apex $O$ (the lengths of the edges of the pyramid are immaterial for the present study, since an arbitrarily small neighborhood of the apex of the pyramid is all that is of concern here). Introduce the cartesian coordinate system in a way that $O A$ makes the same angle with either of the axes $y$ and $z$ as $O B$ does with $z$ and $x$.

* Received March 1, 1977: revised version received May 4, 1977. Support by the Air Force Office of Scientific Research under Grant No. AFOSR 75-2859 is gratefully acknowledged. The authors are also grateful for helpful discussions with Professor Z. P. Bažant during the course of this research.

** Permanent address: Department of Mathematics, Indian Institute of Technology, Bombay, India. 
Then the three planes forming the pyramid are given by

$$
x=(y+z) \cos \alpha, \quad y=(z+x) \cos \alpha, \quad z=(x+y) \cos \alpha
$$

where $\alpha>\pi / 3$. From these equations it is easily seen that

$$
\begin{aligned}
& \cos 2 \beta=\cos \alpha(2-\cos \alpha) /\left(3 \cos ^{2} \alpha-2 \cos \alpha+1\right), \\
& \cos 2 \delta=\cos \alpha(2-\cos \alpha) /\left(1+2 \cos ^{2} \alpha\right),
\end{aligned}
$$

where $2 \delta$ is the angle between any two consecutive planes of the pyramid. From (2)-(3) one obtains the relation (cf. [1, p. 240])

$$
2 \sin \delta \cos \beta=1 .
$$

The potential $\Phi$ due to the surface charge densities $\Delta_{1}, \Delta_{2}$ and $\Delta_{3}$ on the planes $A B$, $B C$, and $C A$ respectively is given by

$$
\Phi=\Phi_{1}+\Phi_{2}+\Phi_{3},
$$

with

$$
\begin{aligned}
& \Phi_{1}(x, y, z)=\iint_{\Omega} \frac{\Delta_{1}(\xi, \eta) d \xi d \eta}{\left[(x-\xi)^{2}+(y-\eta)^{2}+\{z-(\xi+\eta) \cos \alpha\}^{2}\right]^{1 / 2}}, \\
& \Phi_{2}(x, y, z)=\iint_{\Omega} \frac{\Delta_{2}(\eta, \zeta) d \eta d \zeta}{\left[\{x-(\eta+\zeta) \cos \alpha\}^{2}+(y-\eta)^{2}+(z-\zeta)^{2}\right]^{1 / 2}}, \\
& \Phi_{3}(x, y, z)=\iint_{\Omega} \frac{\Delta_{3}(\zeta, \xi) d \zeta d \xi}{\left[(x-\xi)^{2}+\{y-(\zeta+\xi) \cos \alpha\}^{2}+(z-\zeta)^{2}\right]^{1 / 2}},
\end{aligned}
$$

where $\Omega$ denotes the area of projection of the planes $A B, B C$ and $C A$ on the coordinate planes $x y, y z$ and $z x$ respectively. If a constant potential $\tau$ is prescribed on the faces of the pyramid we have

$$
\begin{aligned}
\Phi(\cos \alpha(y+z), y, z) & =\tau, \\
\Phi(x, \cos \alpha(z+x), z) & =\tau, \\
\Phi(x, y, \cos \alpha(x+y)) & =\tau .
\end{aligned}
$$

It is convenient to deal with the above equations in their polar forms. Consider

$$
\begin{aligned}
Q_{1}(x, y) & =\Phi_{1}(x, y, \cos \alpha(x+y)) \\
& =\iint_{\Omega} \frac{\Delta_{1}(\xi, \eta) d \xi d \eta}{\left[(x-\xi)^{2}+(y-\eta)^{2}+\{(x+y)-(\xi+\eta)\}^{2} \cos ^{2} \alpha\right]^{1 / 2}},
\end{aligned}
$$

and introduce the polar transformations

$$
\begin{aligned}
& x=r \cos \theta, \quad y=r \sin \theta \\
& \xi=\rho \cos \phi, \quad \eta=\rho \sin \phi
\end{aligned}
$$

to get

$$
P_{1}(r \cos \theta, r \sin \theta)=\int_{a}^{b} \int_{0}^{\infty} \frac{\Delta_{1}(\rho \cos \phi, \rho \sin \phi) \rho d \rho d \phi}{\left[r^{2} K-2 \rho r L_{1}+\rho^{2} M\right]^{1 / 2}}, \quad a<\theta<b,
$$


where

$$
\begin{gathered}
K=1+2 \cos ^{2} \alpha \cos ^{2}\left(\frac{\pi}{4}-\theta\right), \\
M=1+2 \cos ^{2} \alpha \cos ^{2}\left(\frac{\pi}{4}-\phi\right), \\
L_{1}=\cos (\theta-\phi)+2 \cos ^{2} \alpha \cos \left(\frac{\pi}{4}-\theta\right) \cos \left(\frac{\pi}{4}-\phi\right),
\end{gathered}
$$

and the limits $a, b$ of integration for $\theta, \phi$ are given by

$$
\begin{aligned}
\cos a & =(1-\cos \alpha)\left[(1-\cos \alpha)^{2}+\cos ^{2} \alpha\right]^{-1 / 2}, \\
\cos b & =\cos \alpha\left[(1-\cos \alpha)^{2}+\cos ^{2} \alpha\right]^{-1 / 2} .
\end{aligned}
$$

From these relations it is easily seen that

$$
a+b=\pi / 2 \text {. }
$$

Differentiating (15) with respect to $r$ gives

$$
\frac{\partial}{\partial r} Q_{1}=-\int_{a}^{b} \int_{0}^{\infty} \frac{\Delta_{1}(\rho \cos \phi, \rho \sin \phi)\left(r K-\rho L_{1}\right) \rho d \rho d \phi}{\left[r^{2} K-2 \rho r L_{1}+\rho^{2} M\right]^{3 / 2}}, \quad a<\theta<b .
$$

Then setting (cf. [2])

$$
\Delta_{1}(\rho \cos \phi, \rho \sin \phi)=\rho^{\gamma-1} f_{1}(\phi) \quad(0<\gamma<1),
$$

and making use of the integral (see [3, p. 310])

$$
\int_{0}^{\infty} \frac{x^{s-1} d x}{\left[1+2 x \cos t+x^{2}\right]^{1 / 2}}=\pi \operatorname{cosec}(\pi s) P_{s-1}(\cos t),|t|<\pi, 0<\operatorname{Re} s<1
$$

yields

$$
\frac{\partial}{\partial r} Q_{1}=-K^{\gamma / 2} \frac{\pi \gamma}{\sin \pi \gamma} \int_{a}^{b} M^{-(1+\gamma) / 2} f_{1}(\phi) P_{\gamma}\left(-L_{1} /(K M)^{1 / 2}\right) d \phi, \quad a<\theta<b .
$$

In the same way if one writes, in the notation of (6)-(8) and (13),

$$
Q_{j}(r \cos \theta, r \sin \theta)=\Phi_{j}(x, y, \cos \alpha(x+y)), \quad j=1,2,3
$$

it can be shown that

$$
\begin{aligned}
\frac{\partial}{\partial r} Q_{j} & =-K^{\gamma / 2} \frac{\pi \gamma}{\sin \pi \gamma} \int_{a}^{b} M^{-(1+\gamma) / 2} f_{j}(\phi) P_{\gamma}\left(-L_{j} /(K M)^{1 / 2}\right) d \phi, \\
j & =1,2,3, \quad a<\theta<b,
\end{aligned}
$$

where $K, M$ are given by (16), (17) and we have

$$
\begin{aligned}
& L_{2}=\sin \theta \cos \phi+\sqrt{ } 2 \cos \alpha\left\{\cos \theta \cos \left(\frac{\pi}{4}-\phi\right)+\sin \phi \cos \left(\frac{\pi}{4}-\theta\right)\right\}, \\
& L_{3}=\cos \theta \sin \phi+\sqrt{ } 2 \cos \alpha\left\{\sin \theta \cos \left(\frac{\pi}{4}-\phi\right)+\cos \phi \cos \left(\frac{\pi}{4}-\theta\right)\right\} .
\end{aligned}
$$

In view of relations (5), (26) and (27) the condition (11) becomes 


$$
\begin{array}{r}
\int_{a}^{b}\left[F_{1}(\phi) P_{\gamma}\left\{-A_{0}(\theta, \phi)\right\}+F_{2}(\phi) P_{\gamma}\left\{-B_{0}(\theta, \phi)\right\}+F_{3}(\phi) P_{\gamma}\left\{-C_{0}(\theta, \phi)\right\}\right] d \phi=0, \\
a<\theta<b,
\end{array}
$$

where

$$
\begin{gathered}
F_{j}(\phi)=M^{-(1+\gamma) / 2} f_{j}(\phi), \quad j=1,2,3, \\
A_{0}=L_{1} /(K M)^{1 / 2}, \quad B_{0}=L_{2} /(K M)^{1 / 2}, \quad C_{0}=L_{3} /(K M)^{1 / 2}
\end{gathered}
$$

in which $K, M, L_{j}(j=1,2,3)$ are given by (16)-(18) and (28)-(29). By means of a similar analysis, Eqs. (9)-(10) can be reduced to the same forms as that of (30). Indeed, due to symmetry of the problem the other two equations can be obtained by the cyclic interchange of the unknown functions $F_{j}(\phi)(j=1,2,3)$. Adding all the resulting three equations and setting either $F_{1}=F_{2}=F_{3}=v$ or

$$
F_{1}+F_{2}+F_{3}=v
$$

leads to the eigenvalue equation

$$
\int_{a}^{b} v(\phi)\left[P_{\gamma}\left\{-A_{0}(\theta, \phi)\right\}+P_{\gamma}\left\{-B_{0}(\theta, \phi)\right\}+P_{\gamma}\left\{-C_{0}(\theta, \phi)\right\}\right] d \phi=0, \quad a<\theta<b,
$$

where $a, b$ are given by (19)-(20) and $A_{0}, B_{0}, C_{0}$ by (32), (16)-(18) and (28)-(29). Note the series representation (see e.g. $[4$, Appendix $]$ )

$$
\begin{aligned}
P_{\gamma}(x)= & \frac{1}{\pi} \sin \pi \gamma \sum_{n=0}^{\infty} \frac{(-\gamma)_{n}(1+\gamma)_{n}}{(n !)^{2}}\left[\log \left(\frac{1+x}{2}\right)+\psi(n-\gamma)\right. \\
& +\psi(n+\gamma+1)-2 \psi(n+1)]\left(\frac{1+x}{2}\right)^{n}
\end{aligned}
$$

where $\psi$ denotes the logarithmic derivative of the gamma function (see [5, p. 15]) and

$$
(h)_{0}=1, \quad(h)_{n}=h(h+1) \cdots(h+n-1) .
$$

Then it can be easily seen that all the three terms in the kernel of (34) have logarithmic singularity: the first at $\phi=\theta$, the second at $\theta=b, \phi=a$ and the third at $\theta=a, \phi=b$. Indeed, the leading terms in the kernel of (34) may be written

$$
\begin{aligned}
P_{\gamma}\left(-A_{0}\right)+P_{\gamma}\left(-B_{0}\right)+P_{\gamma}\left(-C_{0}\right) & \simeq 2 \log |\theta-\phi| \\
+ & \log \left\{(b-\phi)^{2}+(\theta-a)^{2}-2(b-\phi)(\theta-a) \cos 2 \delta\right\} \\
+ & \log \left\{(b-\theta)^{2}+(\phi-a)^{2}-2(b-\theta)(\phi-a) \cos 2 \delta\right\},
\end{aligned}
$$

where $2 \delta$, the angle between any two planes of the pyramid, is given by (3). In view of (37), if (34) is differentiated once with respect to $\theta$ it becomes a singular integral equation with a generalized Cauchy kernel discussed by Erdogan, Gupta and Cook [6]. By using an analysis similar to that of [6] one can show that the unknown function $v(\phi)$ in (34) is of the form

$$
v(\phi)=[(b-\phi)(\phi-a)]^{\nu-1} w(\phi),
$$

where $w(\phi)$ is a bounded function on $[a, b]$ and

$$
\nu=\frac{1}{2} \pi /(\pi-\delta)
$$


TABLE 1 .

\begin{tabular}{ccc}
\hline & \multicolumn{2}{c}{$\gamma$} \\
\cline { 2 - 3 } $2 \beta / \pi$ & Integral Equation & Bažant \\
\hline $1 / 32$ & 0.1283 & \\
$1 / 16$ & 0.1557 & \\
$1 / 8$ & 0.1976 & \\
$1 / 4$ & 0.2709 & 0.230 \\
$3 / 8$ & 0.3497 & \\
$1 / 2$ & 0.4534 & 0.455 \\
$5 / 8$ & 0.6580 & \\
$21 / 32$ & 0.8017 & \\
\hline
\end{tabular}

The nature of singularity (39) in the function $v(\phi)$ representing potential on the faces of the pyramid is in agreement with that of Bažant ([1], p. 223).

Numerical solution. The eigenvalue equation (34) does not seem to admit a closedform solution. One can, however, use the procedure of Erdogan, Gupta and Cook [6] to extract the root $\gamma$ for a fixed value of $2 \beta$, the angle between any two consecutive edges of the pyramid. In view of the relations (35)-(37), the differentiation of Eq. (34) once with respect to the free variable leads to a singular integral equation with a generalized Cauchy kernel. Also, the unknown function in (34) has the proper singularity given by (38)-(39) as the end points are approached.

By using the Gauss-Jacobi integration formula for singular integral equations with generalized Cauchy kernels [6], one obtains a system of $N$ homogeneous simultaneous algebraic equations in $N$ unknowns from the differentiated (and appropriately manipulated) Eq. (34). Thus, as in [2] one obtains the eigenvalue problem to be solved numerically by setting the determinant, say, $R_{N}(\beta, \gamma)$ of the coefficient matrix equal to zero, i.e.

$$
R_{N}(\beta, \gamma)=0,
$$

where $2 \beta$ is the angle between any two consecutive edges of the pyramid and $\gamma$ represents the singularity strength at the apex. A numerical root search procedure is applied to Eq. (40) with $N=11$, the number of collocation points. The results of the computation for various $\beta$ are given in Table 1, where Bažant's results are also listed. His computations are based on the finite-difference network of about eighty nodes which are not quite sufficient for the four-digit accuracy presented here. It may be possible to match the present results with a larger-size network using his method.

\section{REFERENCES}

[1] Z. P. Bažant, Three-dimensional harmonic functions near termination or intersection of gradient singularity lines: a general numerical method, Int. J. Engng. Sci. 12, 221-243 (1974)

[2] L. M. Keer and K. S. Parihar, A note on the singularity at the corner of a wedge-shaped punch or crack, SIAM J. Appl. Math., in press.

[3] A. Erdélyi, Tables of integral transforms, Vol. 1, McGraw-Hill, 1954

[4] J. A. Morrison and J. A. Lewis, Charge singularity at the corner of a flat plate, SIAM J. Appl. Math. 31, 233-250 (1976)

[5] A. Erdélyi, Higher transcendental functions, Vol. I, McGraw-Hill, 1953

[6] F. Erdogan, G. D. Gupta and T. S. Cook, Numerical solution of singular integral equations, in Methods of analysis and solutions of crack problems, ed. G. C. Sih, Noordhoff, 1973, pp. 368-425 